\title{
Glucocorticoids induce apoptosis by inhibiting microRNA cluster miR-17-92 expression in chondrocytic cells
}

\author{
WENHUA XING ${ }^{1 *}$, LIXIA HAO ${ }^{2 *}$, XUEJUN YANG ${ }^{1}$, FENG LI $^{1}$ and HONGJUN HUO ${ }^{1}$ \\ ${ }^{1}$ Department of Spinal Surgery, Second Affiliated Hospital of Inner Mongolia Medical University, Huimin, Hohhot, \\ Inner Mongolia Autonomous Region 010058; ${ }^{2}$ Department of Rehabilitation, First Affiliated Hospital of Inner \\ Mongolia Medical University, Huimin, Hohhot, Inner Mongolia Autonomous Region 010059, P.R. China
}

Received September 2, 2013; Accepted March 6, 2014

DOI: $10.3892 / \mathrm{mmr} .2014 .2253$

\begin{abstract}
Sustained treatment with glucocorticoids (GCs) has frequently been observed to impair skeletal development. However, the influence of GCs on chondrocytes, which have a key role in skeletal development, has been rarely reported. HCS-2/8 cells were selected as an in vitro model of human chondrocytes to assess the apoptosis induced by GCs and determine the role of the microRNA-17-92 (miR-17-92) cluster in the regulation of apoptosis. It was demonstrated that dexamethasone (Dex) was able to induce apoptosis and high levels of expression of apoptosis-associated molecules in HCS-2/8 chondrocytic cells, and that expression of the miR-17-92 cluster was inhibited during Dex-induced apoptosis. In conclusion, the present study suggested that inhibition of the expression of the miR-17-92 cluster contributed to the Dex-induced apoptosis in chondrocytes. The results suggest that microRNAs have an important role in glucocorticoid-induced impairment to chondrocytes.
\end{abstract}

\section{Introduction}

Glucocorticoids (GCs) are widely used for the treatment of chronic orthopedic diseases, including lumbar disc herniation, rheumatism and chronic inflammatory or autoimmune diseases. The potent anti-inflammatory and immunosuppressive effects of GCs give them the reputation of being a 'miracle elixir'. However, prolonged treatment with GCs results in alterations in water and salt metabolism, metabolic disorders and impairment of skeletal development (1-3). Sustained administration

Correspondence to: Professor Hongjun Huo, Department of Spinal Surgery, Second Affiliated Hospital of Inner Mongolia Medical University, 1 Yingfang Road, Huimin, Hohhot, Inner Mongolia Autonomous Region 010058, P.R. China

E-mail: huohj2013@163.com

${ }^{*}$ Contributed equally

Key words: glucocorticoids, chondrocytes, apoptosis, microRNA-17-92 cluster of GCs is frequently accompanied by decreased generation of osteoblasts and osteocytes, as well as a prolonged lifespan of osteoclasts. Previous studies have indicated that apoptosis and autophagy in osteocytes may be involved in the mechanism underlying the GC-induced impairment of skeletal development (4-6). The influence of GCs on chondrocytes, which have a key role in skeletal development, has been rarely reported. Certain studies have shown that GCs have an effect on the apoptosis and differentiation of chondrocytes $(7,8)$; however, the details of this require elucidation.

MicroRNAs (miRNAs) are a family of 22 -nucleotide, endogenous, non-coding RNAs and have been identified in organisms ranging from nematodes to humans. miRNAs are able to bind to the $3^{\prime}$ untranslated regions (3'-UTRs) of mRNAs and regulate gene expression by post-transcriptional gene inhibition $(9,10)$. An increasing number of studies have revealed that miRNAs have important roles in the regulation of apoptosis. MicroRNA-708 (miR-708), -195, -206 and -155 have been demonstrated to separately induce or facilitate apoptosis in renal cancer cells, cardiomyocytes, tumor cells and macrophages (11-14). The regulation of apoptosis in chondrocytes and, in particular, its regulation by miRNAs has rarely been studied. However, of note was the finding by Abouheif et al (15) that silencing miR-34a inhibited apoptosis of chondrocytes in a rat osteoarthritis model in vitro. There is a requirement to identify miRNAs that have roles in the regulation of apoptosis in chondrocytes.

In the present study, GC-induced apoptosis was assessed in the human chondrocytic HCS-2/8 cell line and the role of the miR-17-92 cluster in this process was evaluated.

\section{Materials and methods}

Reagents and cell cultures. The GC dexamethasone (Dex, Sigma-Aldrich, St. Louis, MO, USA) was dissolved in $\alpha$-Minimum Essential Medium ( $\alpha$-MEM) with $0.5 \%$ ethanol, and the GC antagonist RU486 (Sigma-Aldrich) was dissolved in dimethylsulfoxide (DMSO). HCS-2/8 cells were provided by the cell resource center of the Chinese Academy of Medical Sciences (Beijing, China). Unless otherwise specified, HCS-2/8 cells were inoculated in Eagle's MEM, supplemented with $20 \%$ fetal bovine serum (FBS) and $0.2 \%$ penicillin/streptomycin at $37^{\circ} \mathrm{C}$ under $5 \% \mathrm{CO}_{2}$. 
Cell viability assay. Cell viability was assessed using the MTT assay. HCS-2/8 cells were seeded in 96-well plates and incubated at $37^{\circ} \mathrm{C}$ for $24 \mathrm{~h}$, prior to the replacement of the medium with Eagle's MEM containing 2\% FBS. Dex $(0,50$ or $250 \mu \mathrm{M})$ and/or RU486 (0 or $250 \mu \mathrm{M})$ was added to the medium and incubation was continued for up to 0,24 or $48 \mathrm{~h}$. The medium was subsequently replaced with $50 \mu \mathrm{l}$ MTT solution and the cells were incubated at $37^{\circ} \mathrm{C}$. After $2 \mathrm{~h}$ of incubation, the MTT solution was discarded and $150 \mu \mathrm{l}$ DMSO was added to completely dissolve the precipitate at room temperature. The optical density was then measured at $570 \mathrm{~nm}$ using a spectrophotometer.

Apoptosis assays. Apoptosis was assessed using an annexin V-fluorescein isothiocyanate (FITC) apoptosis detection kit (Sigma-Aldrich). Briefly, $1-5 \times 10^{5} \mathrm{HCS}-2 / 8$ cells were resuspended in $0.5 \mathrm{ml}$ binding buffer and incubated with annexin V-FITC and propidium iodide for $10 \mathrm{~min}$ in the dark at room temperature. A FACScan flow cytometer (BD Biosciences, Franklin Lanes, NJ, USA) equipped with an FITC signal detector FL1 (excitation $488 \mathrm{~nm}$, green) and a phycoerythrin emission signal detector FL3 (excitation $585 \mathrm{~nm}$, red) was used to quantify cellular apoptosis. The results were calculated using the CellQuest ${ }^{\mathrm{TM}}$ Pro software (BD Biosciences) and expressed as the percentage of apoptotic cells among the total cells.

RNA isolation, quantitative polymerase chain reaction ( $q P C R$ ) and RNase protection assay. Total cellular RNA from $\sim 10^{5}$ cells was prepared using TRIzol ${ }^{\circledR}$ reagent (Invitrogen Life Technologies, Carlsbad, CA, USA). miRNAs were isolated using the mirVANA ${ }^{\mathrm{TM}}$ miRNA isolation kit (Ambion, Austin, TX, USA). qPCR was performed with the Takara One-Step RT-PCR kit (Takara Bio, Inc., Dalian, China). For quantitative analysis of the mRNA expression of BCL2-associated X protein (Bax), caspase 3, Drosha and Dicer, the resulting cDNAs were amplified using primer/probe sets specific for the genes of interest on a Lightcycler 480 II (Roche, Mannheim, Germany). Relative quantification was performed using the $\Delta \Delta \mathrm{Ct}$ method with GAPDH as the reference gene. The primer sequences used in the present study are shown in Table I.

Western blot analysis. Whole cell lysates were obtained by suspending $\sim 10^{5}$ cells in cell lysis reagent (Promega Corp., Madison, WI, USA), and Complete Mini Protease Inhibitor Cocktail (Roche). Following protein concentration determination using Bradford Reagent (Bio-Rad, Hercules, CA, USA), equal amounts of protein and sample buffer were separated using 4-20\% gradient SDS-PAGE, transferred onto a polyvinylidene fluoride membrane and blocked in Tris-buffered saline containing skimmed milk (5\%). The following primary antibodies were used: Caspase 3 rabbit polyclonal antibody, 1:500 (Sigma-Aldrich); Dicer rabbit polyclonal antibody, 1:500 (Santa Cruz Biotechnology, Inc., Santa Cruz, CA, USA); Drosha rabbit polyclonal antibody, 1:500 (Santa Cruz Biotechnology, Inc.); and GAPDH rabbit polyclonal antibody, 1:1,000 (Sigma-Aldrich). All immunoblots are representative of at least three independent experiments.

Overexpression of miR-17-92. Overexpression of miR-17-92 was performed using miRNA mimics (Qiagen, Valencia, CA, USA) for individual miRNAs: miR-17, miR-18, miR-19a and miR-92.
For the assessment of the association between miR-17-92 and apoptosis, a mixture of all four miRNA mimics ( $200 \mathrm{nM}$ each) or $1,200 \mathrm{nM}$ nontargeting control was transfected into HCS-2/8 cells with Lipofectamine 2000. Six hours post-transfection, cells were incubated with Dex for 8 or $24 \mathrm{~h}$ and assayed for apoptosis.

Statistical analysis. Statistical analyses were performed using SPSS 16.0 software (SPSS, Inc., Chicago, IL, USA). The differences in cell viability, apoptosis, expression of Cas3, Bax, miRNAs, Drosha or Dicer between the two groups were analyzed by Student's t test. $\mathrm{P}<0.05$ was to indicate a statistically significant difference.

\section{Results}

Dex reduces HCS-2/8 chondrocyte cell viability via the induction of apoptosis. To assess the effect of Dex on the viability of HCS-2/8 chondrocytic cells, the MTT assay was conducted. Fig. 1A shows that Dex was able to reduce the cell viability following $24 \mathrm{~h}(82.51 \pm 5.60 \%, \mathrm{P}<0.05$ versus the control at $50 \mu \mathrm{M} ; 72.00 \pm 2.85 \%, \mathrm{P}<0.01$ versus the control at $250 \mu \mathrm{M})$ or $48 \mathrm{~h}(72.30 \pm 5.91 \%, \mathrm{P}<0.01$ versus the control at $50 \mu \mathrm{M}$; $59.63 \pm 4.90 \%, \mathrm{P}<0.01$ versus the control at $250 \mu \mathrm{M})$ of incubation. The GC antagonist RU486 $(50 \mu \mathrm{M})$ was utilized to re-confirm the effect of Dex on cell viability. HCS-2/8 cells were pre-treated with RU486 prior to incubation with Dex. It was shown that RU486 reversed the decrease in cell viability induced by Dex after $48 \mathrm{~h}$ of incubation (Fig. 1B).

$\mathrm{GC}$-induced apoptosis has been reported in various cell models (16-19), including chondrocyte cells $(20,21)$; therefore, it was assessed whether the decreased cell viability following Dex treatment was due to apoptosis. Fig. 1C-E shows that apoptosis was significantly increased following incubation with Dex $(250 \mu \mathrm{M})$ for 8 or $24 \mathrm{~h}(\mathrm{P}<0.01)$. To further confirm that apoptosis was induced by Dex, the mRNA and protein expression levels of the proapoptotic Bax and the apoptosis executioner caspase 3 were also assayed by qPCR and western blot analysis. mRNA (Fig. 2A and B) and protein levels (Fig. 2C and D) of Bax and caspase 3 in HCS-2/8 cells were significantly higher following incubation with Dex. In addition, the GC antagonist RU486 was able to inhibit the Dex-induced expression of apoptosis-associated molecules (Fig. 2A and B).

Inhibition of miR-17-92 cluster expression and miRNA processing enzymes during GC-induced apoptosis in HCS-2/8 chondrocytic cells. To date, the knowledge on the mechanism underlying Dex-induced apoptosis in chondrocyte cells is limited. An increasing number of studies have shown that miRNAs are involved in apoptosis $(22,23)$; in particular, the GC-mediated inhibition of the expression of the miR-17-92 cluster has been shown to contribute to the initiation of apoptosis in a T-cell lymphoma cell line (24). To evaluate the possible role of the miR-17-92 cluster in Dex-induced apoptosis in HCS-2/8 chondrocytic cells, qPCR was used to determine the expression of miR-17,-18,-19a and -92. Fig. 3A and B shows that these four miRNAs were downregulated in $\mathrm{HCS}-2 / 8$ cells following incubation with $250 \mu \mathrm{M}$ Dex for $12 \mathrm{~h}(\mathrm{P}<0.05$ versus the control); however, this was not observed in the $50 \mu \mathrm{M}$ Dex treatment group ( $\mathrm{P}>0.05$ versus the control). To further investigate the downregulation of the mature miR-17-92 cluster by 
Table I. Primer sequences for quantitative polymerase chain reaction.

\begin{tabular}{lll}
\hline Gene & \multicolumn{1}{c}{ Forward primer $\left(5^{\prime}\right.$ to $\left.3^{\prime}\right)$} & \multicolumn{1}{c}{ Reverse primer $\left(5^{\prime}\right.$ to $\left.3^{\prime}\right)$} \\
\hline Bax & TGG AGCTGCAGAGGATGATTG & GAAGTTGCCGTCAGAAAACATG \\
Cas3 & TTC ATT ATT CAG GCC TGC CGA GG & TTC TGA CAG GCC ATG TCA TCC TCA \\
Dicer & TTAACCTTTTGGTGTTGATGAGTGT & GCGAGGACATGATGGACAATT \\
Drosha & TAGGCTGTGGGAAAGGACCAAG & GTTCGATGAACCGCTTCTGATG \\
GAPDH & AATCCCATCACCATCTTCCA & TGGACTCCACGACGTACTCA
\end{tabular}

Bax, BCL2-associated X protein; Cas3, caspase-3.

A

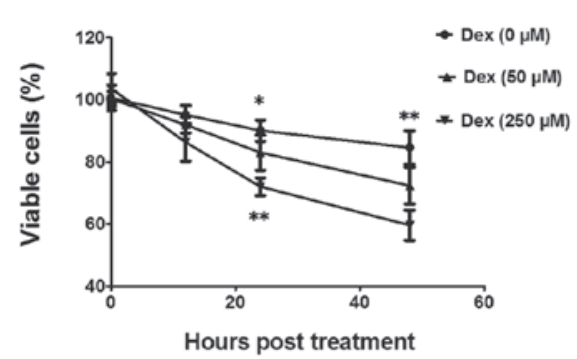

B

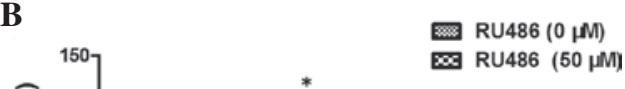

C
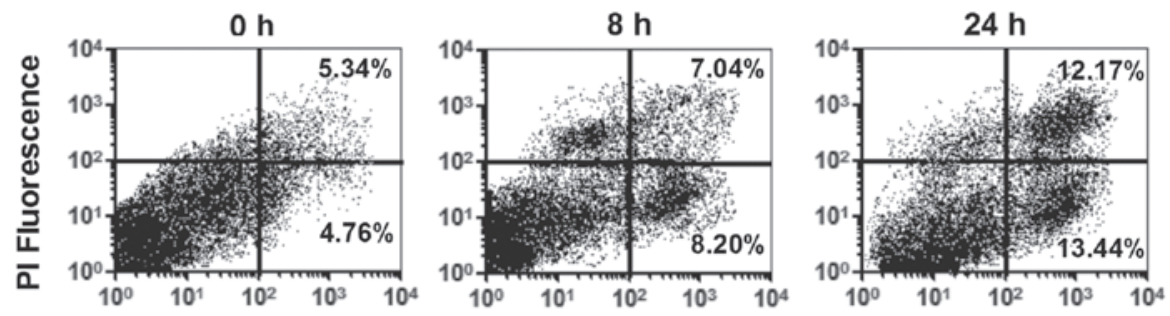

Annexin-FITC Fluorescence

D
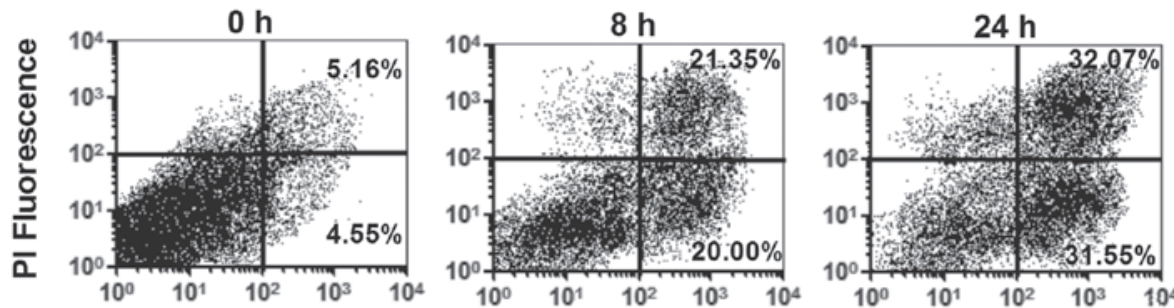

$\mathbf{E}$

Annexin-FITC Fluorescence

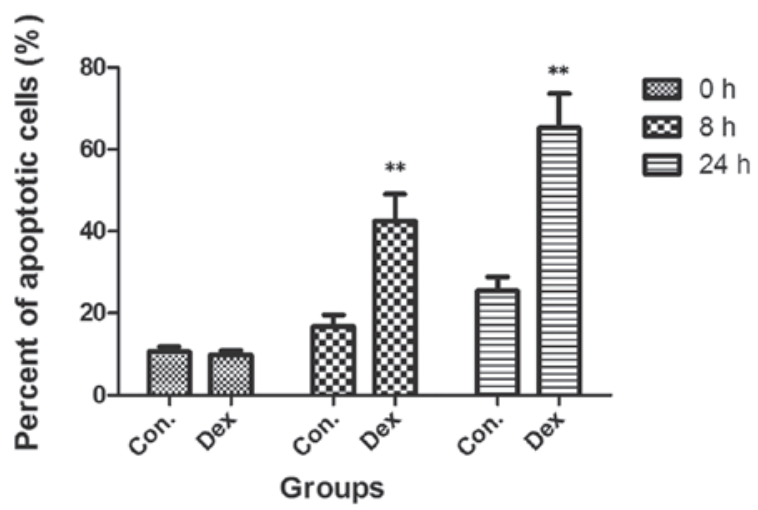

Figure 1. Dex reduces viability and induces apoptosis in HCS-2/8 cells. (A) Viability of HCS-2/8 cells 12,24 or 48 h post-treatment with 0,50 or $250 \mu \mathrm{M}$ Dex. (B) RU486 blocks the HCS-2/8 cell viability decrease induced by Dex. HCS-2/8 cells were pretreated with 0 or $50 \mu$ M RU486 and incubated with Dex ( $0,10,50$ or $250 \mu \mathrm{M}$ ) for $48 \mathrm{~h}$. Cell viability was determined using the MTT assay. (C) Apoptosis of HCS-2/8 cells not treated with Dex. (D) Apoptosis of HCS-2/8 cells incubated with Dex $(250 \mu \mathrm{M})$. (E) Quantification of apoptosis of HCS-2/8 cells with or without Dex treatment. A significant difference was observed in levels of apoptosis between HCS-2/8 cells with and without Dex treatment. Results are expressed as percentages of positive mean values \pm standard error for three independent experiments. ${ }^{*} \mathrm{P}<0.05 ;{ }^{* *} \mathrm{P}<0.01$. Dex, dexamethasone; FITC, fluorescein isothiocyanate; PI, propidium iodide; Con, control. 
A

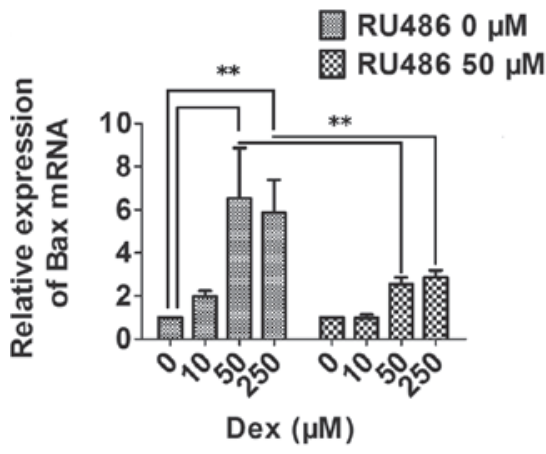

C

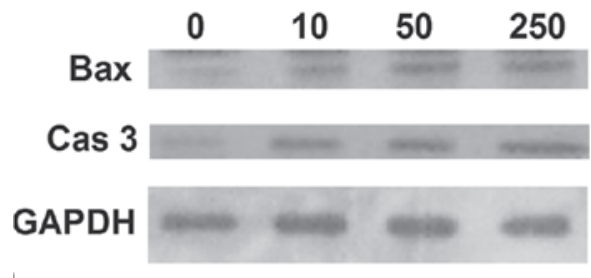

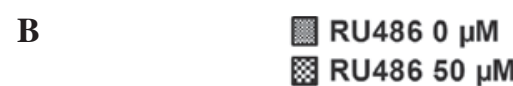

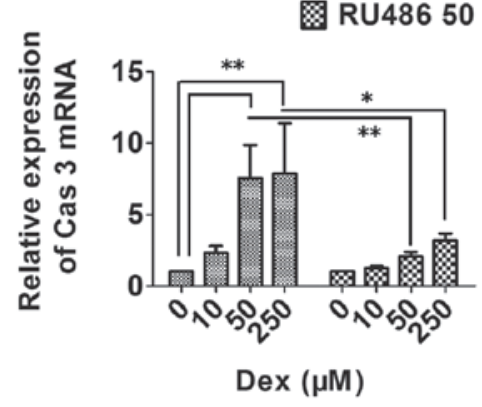

D

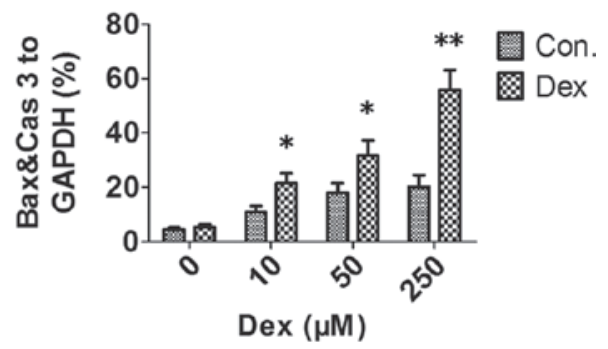

Figure 2. Dex induces high expression levels of apoptosis-associated molecules in HCS-2/8 cells. (A and B) Bax and caspase 3 mRNA expression in HCS-2/8 cells was evaluated by the quantitative polymerase chain reaction and normalized to the GAPDH housekeeping gene. HCS-2/8 cells were lysed following incubation with RU486 and/or Dex for $12 \mathrm{~h}$ for the assessment of mRNA expression. mRNA expression was assessed in relation to GAPDH. (C and D) HCS-2/8 cells were lysed post-incubation with Dex for $24 \mathrm{~h}$ for western blot analysis. Whole cell lysates were immunoblotted with Bax or caspase 3 polyclonal antibodies. GAPDH immunoblotting served as a loading control. All results are representative of at least three independent experiments. ${ }^{*} \mathrm{P}<0.05$; ${ }^{* *} \mathrm{P}<0.01$. Bax, BCL-2 associated X protein; Cas 3; caspase 3; Dex, dexamethasone; Con, control.

GCs, the expression of the key miRNA processing enzymes Drosha and Dicer was assessed during GC-induced apoptosis of HCS-2/8 cells. Western blot analysis and qPCR demonstrated that the expression of Drosha and Dicer was inhibited by Dex at the mRNA and protein levels (Fig. 3C-F).

Overexpression of the miR-17-92 cluster inhibits $G C$-induced apoptosis. To reconfirm the potential anti-apoptotic role of the miR-17-92 cluster, which was downregulated during GC-induced apoptosis in HCS-2/8 cells, the miR-17-92 cluster was overexpressed in HCS-2/8 cells via transfection with the hsa-miR-17-92 miRNA mimics miR-17, miR-18, miR-19a and miR-92. Overexpression of the hsa-miR-17-92 cluster in the HCS-2/8 cells significantly reduced the ability of Dex to induce apoptosis (Fig. 4A and B; Fig. 1C and E). This reduced sensitivity was not due to decreased GC receptor (GR) expression in response to hsa-miR-17-92 overexpression (data not shown). These data suggest that the specific inhibition of the miR-17-92 cluster contributes to the GC-induced apoptosis of chondrocyte cells in vitro.

\section{Discussion}

Chondrocytes have a key role in skeletal development, and studies have shown that GCs exert an effect on the apoptosis and differentiation of chondrocytes $(7,8)$. The present study demonstrated that Dex induced apoptosis in HCS-2/8 cells and reduced cell viability. Dex also inhibited the expression of the proapoptotic protein Bax and the apoptosis executioner caspase 3. The GC antagonist RU486 was able to inhibit the decrease in cell viability and the high expression of Bax and caspase 3 mRNA induced by Dex. Therefore, the apoptosis and decrease in cell viability induced by Dex is GR-dependent.

The mechanism underlying GC-induced apoptosis in chondrocytes has yet to be fully elucidated; however, the mechanism of GC-driven apoptosis in lymphoid cells has been investigated to a greater extent. The participation of effector caspases, including caspase 3, the prosurvival role of B-cell lymphoma 2 (25) and the direct interactions between the GR and the transcription factors nuclear factor of $\kappa$ light polypeptide gene enhancer in B cells and activator protein 1 have been documented (26). Additional signaling molecules, including related adhesion focal tyrosine kinase, signal transducer and activator of transcription 3, calcium and interleukin 6 have also been identified to participate in the GC-induced apoptotic signaling cascade (27-30). However, the knowledge of these signaling molecules and signaling events is currently not sufficient to establish a logical chronology. Therefore, further studies are required to define the signaling pathways involved in GC-induced apoptosis.

Noncoding miRNAs have emerged as important gene expression regulatory elements $(31,32)$. Transcribed by RNA polymerase II, miRNA precursors undergo extensive post-transcriptional processing by the nuclear RNase III enzyme Drosha and the RNase III enzyme Dicer in the cytoplasm. The biologically active, mature, single-stranded miRNA is then incorporated into the miRNA-induced silencing complex to affect gene expression through the inhibitory engagement of complementary 'seed sequences' within the 3'-UTRs of target mRNAs, resulting in translational inhibition $(33,34)$. Through the modulation of gene expression, miRNAs are key effectors of fundamental biological processes, including development, differentiation and apoptosis (35). It has been demonstrated that the miR-17-92 cluster 
A

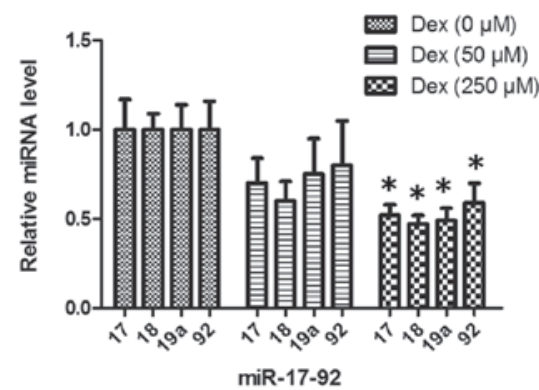

D
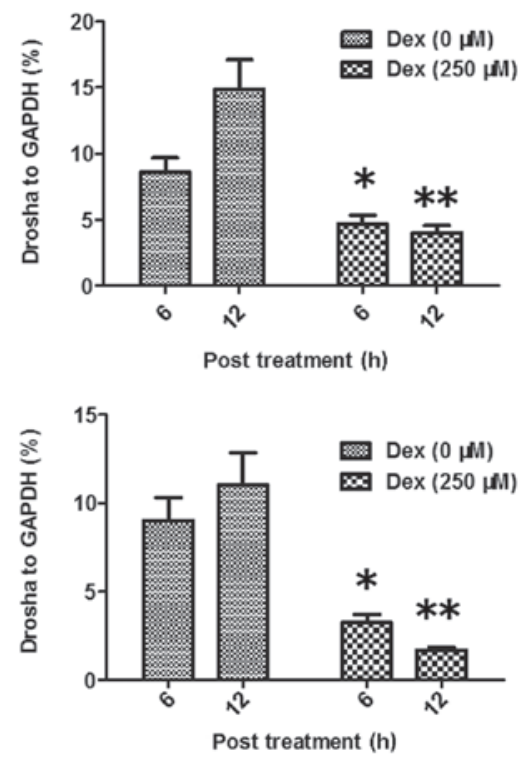

B

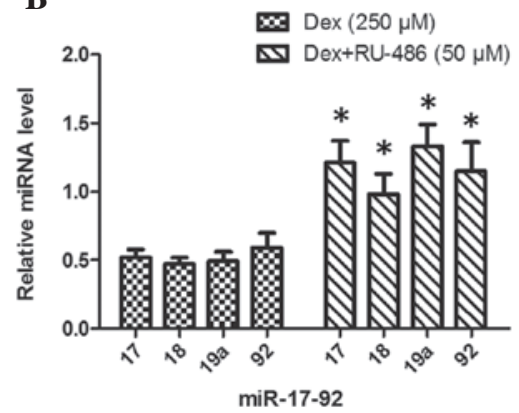

E

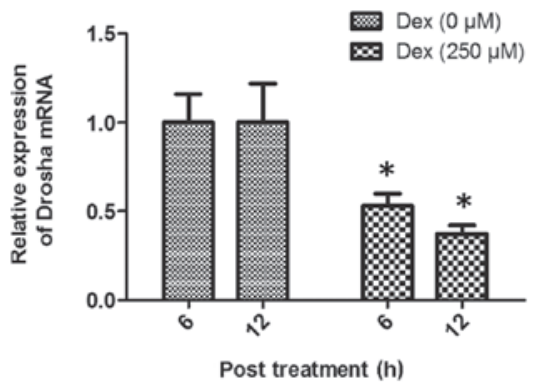

C

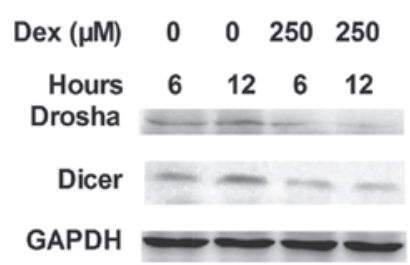

F

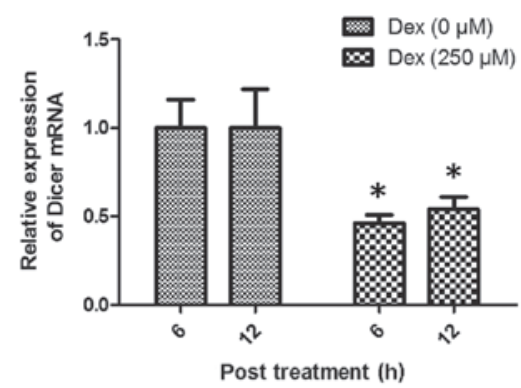

Figure 3. Dex inhibits the expression of the miR-17-92 cluster and miRNA processing enzymes in HCS-2/8 cells. (A and B) qPCR of miR-17, $-18 \mathrm{a},-19$ and -92 in HCS-2/8 cells incubated with increasing concentrations of Dex and RU486 for $24 \mathrm{~h}$. (C and D) HCS-2/8 cells were treated with or without $250 \mu \mathrm{M}$ Dex for 6 and $12 \mathrm{~h}$ prior to the assessment of the expression of the miRNA processing enzymes Drosha and Dicer; whole cell lysates were immunoblotted with Drosha or Dicer polyclonal antibodies. GAPDH immunoblotting served as a loading control. (E and F) mRNA expression of Drosha and Dicer was evaluated using qPCR and normalized to GAPDH. All results are expressed as the mean \pm standard error for three independent experiments. ${ }^{*} \mathrm{P}<0.05$, ${ }^{* *} \mathrm{P}<0.01$ versus untreated cells. qPCR, quantitative polymerase chain reaction; Dex, dexamethasone; miR-17-92, microRNA-17-92.

A
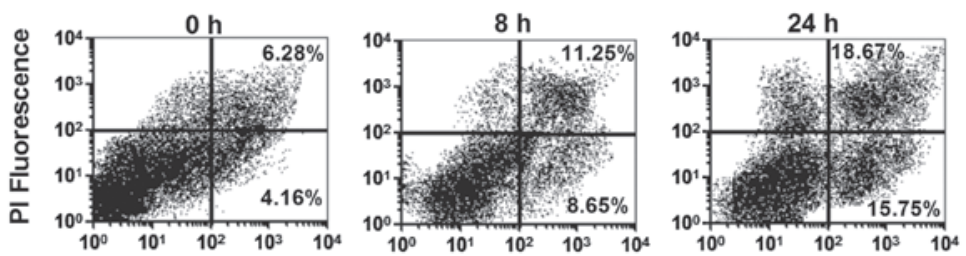

Annexin-FITC Fluorescence

B

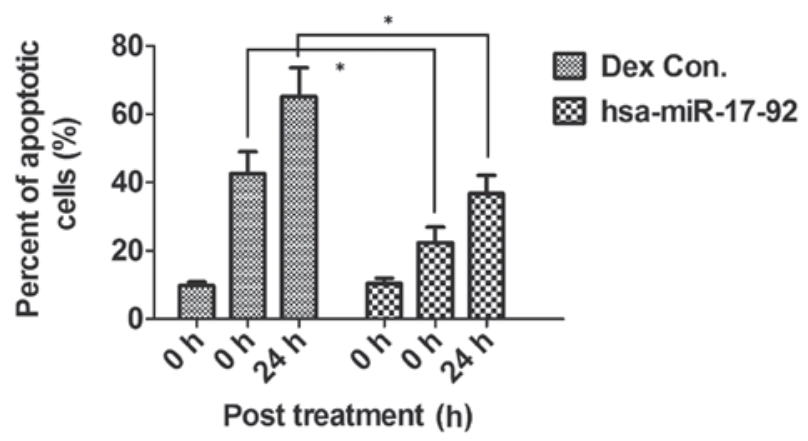

Figure 4. Overexpression of the miR-17-92 cluster inhibits Dex-induced apoptosis in HCS-2/8 cells. (A) Dex-induced apoptosis of HCS-2/8 cells with miR-17-92 cluster overexpression. (B) Significant difference in Dex-induced apoptosis between HCS-2/8 cells with and without miR-17-92 cluster overexpression. Results are expressed as percentages of positive mean values \pm standard error for three independent experiments. " $\mathrm{P}<0.05$. Dex, dexamethasone; FITC, fluorescein isothiocyanate; PI, propidium iodide; Con, control; miR-17-92, microRNA-17-92. 
possesses a conserved anti-apoptotic function in lymphocytes in vitro and in vivo $(24,36-38)$ by inhibiting the expression of the proapoptotic Bcl-2-interacting mediator of cell death 2 as well as that of the phosphatase and tensin homolog (38). It remains to be elucidated whether the miR-17-92 cluster is involved in the apoptosis of chondrocytes following treatment with GCs.

In the present study, HCS-2/8 cells were selected as an in vitro model of human chondrocytes to evaluate the apoptosis induced by Dex, and the possible role of the miR-17-92 cluster in the regulation of apoptosis was investigated. It was demonstrated that Dex was able to inhibit the expression of the miR-17-92 cluster, and this was accompanied by a reduced expression of the miRNA-processing enzymes Drosha and Dicer. The modulation of miR-17-92 expression altered GC-induced apoptosis: Overexpression of miR-17-92 partially inhibited apoptosis in HCS-2/8 cells.

In conclusion, the present study demonstrated that Dex was able to induce apoptosis in HCS-2/8 chondrocytic cells, and that the expression of the miR-17-92 cluster was inhibited during Dex-induced apoptosis. It was indicated that inhibition of the expression of the miR-17-92 cluster contributed to the Dex-induced apoptosis in chondrocytes. The results suggest that microRNAs have an important role in glucocorticoid-induced impairment to chondrocytes.

\section{Acknowledgements}

The present study was supported by grants from the Second Affiliated Hospital of Inner Mongolia Medical University (Hohhot, China).

\section{References}

1. Canalis E and Delany AM: Mechanisms of glucocorticoid action in bone. Ann NY Acad Sci 966: 73-81, 2002.

2. Altman A, Hochberg Z and Silbermann M: Interactions between growth hormone and dexamethasone in skeletal growth and bone structure of the young mouse. Calcif Tissue Int 51: 298-304, 1992.

3. Allen DB: Growth suppression by glucocorticoid therapy. Endocrinol Metab Clin North Am 25: 699-717, 1996.

4. Weinstein RS, Nicholas RW and Manolagas SC: Apoptosis of osteocytes in glucocorticoid-induced osteonecrosis of the hip. J Clin Endocrinol Metab 85: 2907-2912, 2000.

5. Xia X, Kar R, Gluhak-Heinrich J, et al: Glucocorticoid-induced autophagy in osteocytes. J Bone Miner Res 25: 2479-2488, 2010.

6. Jia J, Yao W, Guan M, et al: Glucocorticoid dose determines osteocyte cell fate. FASEB J 25: 3366-3376, 2011.

7. Owen HC, Roberts SJ, Ahmed SF and Farquharson C: Dexamethasone-induced expression of the glucocorticoid response gene lipocalin 2 in chondrocytes. Am J Physiol Endocrinol Metab 294: E1023-E1034, 2008.

8. Mushtaq T, Farquharson C, Seawright E and Ahmed SF: Glucocorticoid effects on chondrogenesis, differentiation and apoptosis in the murine ATDC5 chondrocyte cell line. J Endocrinol 175: 705-713, 2002.

9. Bartel DP: MicroRNAs: target recognition and regulatory functions. Cell 136: 215-233, 2009.

10. Shukla GC, Singh J and Barik S: MicroRNAs: Processing, maturation, target recognition and regulatory functions. Mol Cell Pharmacol 3: 83-92, 2011.

11. Saini S, Yamamura S, Majid S, et al: MicroRNA-708 induces apoptosis and suppresses tumorigenicity in renal cancer cells Cancer Res 1: 6208-6219, 2011.

12. Zhu H, Yang Y, Wang Y, et al: MicroRNA-195 promotes palmitate-induced apoptosis in cardiomyocytes by down-regulating Sirt1. Cardiovasc Res 92: 75-84, 2011.

13. Song G, Zhang Y and Wang L: MicroRNA-206 targets notch3, activates apoptosis, and inhibits tumor cell migration and focus formation. J Biol Chem 284: 31921-3127, 2009.
14. Ghorpade DS, Leyland R, Kurowska-Stolarska M, Patil SA and Balaji KN: MicroRNA-155 is required for Mycobacterium bovis BCG-mediated apoptosis of macrophages. Mol Cell Biol 32: 2239-2253, 2012.

15. Abouheif MM,Nakasa T,Shibuya H, etal: Silencing microRNA-34a inhibits chondrocyte apoptosis in a rat osteoarthritis model in vitro. Rheumatology (Oxford) 49: 2054-2060, 2010.

16. Dowd DR, MacDonald PN, Komm BS, et al: Evidence for early induction of calmodulin gene expression in lymphocytes undergoing glucocorticoid-mediated apoptosis. J Biol Chem 266: 18423-18426, 1991.

17. Carey KT, Tan KH, Ng J, et al: Nfil3 is a glucocorticoid-regulated gene required for glucocorticoid-induced apoptosis in male murine T cells. Endocrinology 154: 1540-1552, 2013.

18. Talabér G, Boldizsár F, Bartis D, et al: Mitochondrial translocation of the glucocorticoid receptor in double-positive thymocytes correlates with their sensitivity to glucocorticoid-induced apoptosis. Int Immunol 21: 1269-1276, 2009.

19. Jewell CM, Scoltock AB, Hamel BL, Yudt MR and Cidlowski JA: Complex human glucocorticoid receptor dim mutations define glucocorticoid induced apoptotic resistance in bone cells. Mol Endocrinol 26: 244-256, 2012.

20. Zaman F, Chrysis D, Huntjens K, Fadeel B and Sävendahl L: Ablation of the pro-apoptotic protein Bax protects mice from glucocorticoid-induced bone growth impairment. PLoS One 7: e33168, 2012.

21. Chrysis D, Zaman F, Chagin AS, Takigawa M and Sävendahl L: Dexamethasone induces apoptosis in proliferative chondrocytes through activation of caspases and suppression of the Akt-phosphatidylinositol 3'-kinase signaling pathway. Endocrinology 146: 1391-1397, 2005.

22. Su H, Yang JR, Xu T, et al: MicroRNA-101, down-regulated in hepatocellular carcinoma, promotes apoptosis and suppresses tumorigenicity. Cancer Res 69: 1135-1142, 2009

23. Carletti MZ, Fiedler SD and Christenson LK: MicroRNA 21 blocks apoptosis in mouse periovulatory granulosa cells. Biol Reprod 83: 286-295, 2010.

24. Molitoris JK, McColl KS and Distelhorst CW: Glucocorticoid-mediated repression of the oncogenic microRNA cluster miR-17 92 contributes to the induction of Bim and initiation of apoptosis. Mol Endocrinol 25: 409-420, 2011.

25. Kofler R: The molecular basis of glucocorticoid-induced apoptosis of lymphoblastic leukemia cells. Histochem Cell Biol 114: 1-7, 2000.

26. Greenstein S, Ghias K, Krett NL and Rosen ST: Mechanisms of glucocorticoid-mediated apoptosis in hematological malignancies. Clin Cancer Res 8: 1681-1694, 2002.

27. Chauhan D, Hideshima T, Pandey P, et al: RAFTK/PYK2-dependent and -independent apoptosis in multiple myeloma cells. Oncogene 18: 6733-6740, 1999.

28. Epling-Burnette PK, Liu JH, Catlett-Falcone R, et al: Inhibition of STAT3 signaling leads to apoptosis of leukemic large granular lymphocytes and decreased Mcl-1 expression. J Clin Invest 107: 351-362, 2001.

29. Distelhorst CW and Dubyak G: Role of calcium in glucocorticosteroid-induced apoptosis of thymocytes and lymphoma cells: resurrection of old theories by new findings. Blood 91: 731-734, 1998.

30. Chauhan D, Pandey P, Ogata A, et al: Dexamethasone induces apoptosis of multiple myeloma cells in a JNK/SAP kinase independent mechanism. Oncogene 15: 837-843, 1997.

31. Bartel DP: MicroRNAs: Target recognition and regulatory functions. Cell 136: 215-233, 2009

32. Brennecke J, Hipfner DR, Stark A, Russell RB, Cohen SM: Bantam encodes a developmentally regulated microRNA that controls cell proliferation and regulates the proapoptotic gene hid in drosophila. Cell 113: 25-36, 2003.

33. Kim VN: MicroRNA biogenesis: coordinated cropping and dicing. Nat Rev Mol Cell Biol 6: 376-385, 2005.

34. Cullen BR: Transcription and processing of human microRNA precursors. Mol Cell 16: 861-865, 2004.

35. Esquela-Kerscher A and Slack FJ: Oncomirs - microRNAs with a role in cancer. Nat Rev Cancer 6: 259-269, 2006

36. He L, Thomson JM, Hemann MT, et al: A microRNA polycistron as a potential human oncogene. Nature 435: 828-833, 2005.

37. Xiao C, Srinivasan L, Calado DP, et al: Lymphoproliferative disease and autoimmunity in mice with increased miR-17-92 expression in lymphocytes. Nat Immunol 9: 405-414, 2008.

38. Smith LK, Shah RR and Cidlowski JA: Glucocorticoids modulate microRNA expression and processing during lymphocyte apoptosis. J Biol Chem 285: 36698-36708, 2010. 